\title{
Avaliação radiográfica e tomográfica de cães submetidos à artroplastia coxofemoral total híbrida
}

\author{
[Radiographic and tomographic evaluation of total hybrid hip replacement in dogs] \\ B.W. Minto ${ }^{1}$, C.V.S. Brandão ${ }^{2}$, G.J.C. Pereira ${ }^{2}$, V.R. Babicsak ${ }^{2}$, \\ L.C. Vulcano ${ }^{2}$,V.J.V. Rossetto ${ }^{2}$ \\ ${ }^{1}$ Unesp - Jaboticabal, SP \\ ${ }^{2}$ Unesp - Botucatu, SP
}

\begin{abstract}
RESUMO
A artroplastia total da articulação coxofemoral é a técnica cirúrgica mais efetiva para o tratamento da displasia coxofemoral canina grave. Na atualidade, o exame radiográfico é o método mais utilizado para se avaliar esse procedimento em cães, porém possui algumas limitações. Já o exame tomográfico por meio de tomografia computadorizada possibilita a aquisição de imagens sem sobreposições e, dessa forma, a determinação do posicionamento dos componentes protéticos com maior precisão. A presente pesquisa teve como objetivo avaliar, por meio de exames radiográficos e tomográficos, a utilização de uma prótese total modular híbrida da articulação coxofemoral desenvolvida e confeccionada no Brasil, aplicada experimentalmente em cães sadios. Foram utilizados seis cães esqueleticamente maduros, previamente submetidos ao procedimento cirúrgico da articulação coxofemoral esquerda. Os cães foram submetidos ao exame tomográfico aos 360 dias e três anos após a cirurgia. Todos os animais apresentaram bom posicionamento dos componentes protéticos e redução articular adequada. Ao exame tomográfico, entretanto, foram detectadas áreas de preenchimento irregular em alguns animais. Adicionalmente, o exame tomográfico permitiu a deteç̧ão e monitoração de áreas de reabsorção óssea ao redor do componente acetabular. Assim, o exame tomográfico mostrou-se útil na avaliação das articulações protéticas, com a vantagem de apresentar ótima definição das imagens e permitir quantificar alterações periprotéticas e mensurar relações articulares, fundamentais para o acompanhamento póscirúrgico tardio.
\end{abstract}

Palavras-chave: canino, diagnóstico por imagem, prótese, coxofemoral

\begin{abstract}
The total hip arthroplasty is the most effective surgical technique for the treatment of severe canine hip dysplasia. Currently, the radiographic examination is the most common method used to assess this procedure in dogs, but has some limitations. CT scan by computed tomography instead enables the acquisition of images without overlap, thereby determining the positioning of prosthetic components with greater accuracy. This research aimed to evaluate by radiography and CT scan examinations the use of a hybrid modular total hip prosthesis developed and made in Brazil, applied experimentally in healthy dogs. Six skeletally mature dogs, which previously underwent surgery on the left hip joint were used. The dogs underwent CT scan at 360 days and three years after surgery. All animals showed good positioning of prosthetic components, and proper joint reduction. In the CT scan, however, it was possible to detect irregular fill areas in some animals. In addition, CT scan allowed the detection and monitoring of areas of bone resorption around the acetabular component. Therefore, the CT scan was useful in the evaluation of prosthetic joints, with the advantage of optimum sharpness of the images and allowing for the possibility of quantifying peri-prosthetic changes and measure joint, fundamental relations for late postoperative follow-up.
\end{abstract}

Keywords:canine, diagnostic imaging, prostheses, hip

Recebido em 29 de setembro de 2015

Aceito em 20 de abril de 2016

E-mail: brunowminto@gmail.com 


\section{INTRODUÇÃO}

A artroplastia total da articulação coxofemoral representa uma das técnicas mais indicadas para o tratamento cirúrgico da displasia coxofemoral canina grave (Lascelles et al., 2010) e refere-se à substituição das extremidades ósseas que compõem a articulação coxofemoral (Minto et al., 2011).

A avaliação radiográfica pós-operatória é comumente utilizada em humanos para a investigação de diversas alterações, tais como migração dos implantes, fraturas, reações periosteais, remodelação óssea proximal adaptativa, reabsorção óssea, debris metálicos e mineralizações distróficas. No entanto, a ocorrência de algumas anormalidades causadoras de dor pós-operatória, tais como infecções, metaloses e soltura asséptica dos implantes, pode não ser detectada ao exame radiográfico em estágios iniciais (Chang et al., 2015; Goldvasser et al., 2012). Nesses casos, a tomografia computadorizada, uma técnica que proporciona imagens multiplanares sem sobreposições e análises tridimensionais (Goldvasser et al., 2012), pode ser considerada um importante método de imagem, complementar à avaliação radiográfica.

Não foram encontrados estudos com a aplicação do sistema protético híbrido para o tratamento da displasia coxofemoral canina, assim como a utilização do exame tomográfico para a sua avaliação na literatura nacional. Tal fato, associado à indicação do procedimento para uma afecção cada vez mais frequente na clínica de pequenos animais, ao alto custo da prótese importada e à pouca difusão da técnica no Brasil, estimulou o desenvolvimento da presente pesquisa.

O objetivo do presente estudo foi avaliar a evolução pós-operatória tardia de uma prótese total modular híbrida da articulação coxofemoral desenvolvida e confeccionada no Brasil, experimentalmente aplicada em cães sadios, por meio de estudos radiográficos e tomográficos, bem como avaliar ambas as técnicas quanto as suas vantagens e limitações.

\section{MATERIAL E MÉTODOS}

Todos os procedimentos utilizados foram aprovados pela Câmara de Ética em Experimentação, sob protocolo 205/2010 CEUA.

Foram utilizados seis animais da espécie canina, hígidos e sem alterações ortopédicas, previamente submetidos ao procedimento de prótese total modular híbrida da articulação coxofemoral esquerda. A prótese foi composta por um componente femoral cimentado de cabeça intercambiável, com possibilidade de seleção de dois diferentes diâmetros de cabeça femoral e três comprimentos de colo femoral, e um componente acetabular não cimentado, subdividido em uma taça metálica de cromocobalto revestida externamente por uma rede de fibras de titânio, um subcomponente de polietileno de alta densidade que se fixa ao primeiro. A taça metálica foi fixada primariamente ao leito ósseo previamente preparado com auxílio de dois ou três parafusos corticais, direcionados aos ossos ílio e ísquio, a partir do leito acetabular.

Todos os animais foram submetidos aos exames radiográficos e tomográficos das articulações coxofemorais aos 360 dias (M1) e três anos (M2) após o procedimento cirúrgico inicial.

Aos exames radiográfico e tomográfico, foi realizada a projeção ventrodorsal, na qual os animais foram posicionados em decúbito dorsal com os membros pélvicos estendidos caudalmente, até extensão completa das articulações femorotibiopatelares. Os membros pélvicos foram mantidos paralelos entre si e rotacionados medialmente, centralizando as patelas no sulco troclear. A pelve foi mantida sem rotação, de forma que os forames obturadores fossem visibilizados de forma simétrica.

A técnica empregada no exame radiográfico variou em torno de 6 a 12 miliamperes $(\mathrm{mA})$ e de 65 a 75 quilovolts (Kvp), de acordo com o porte do animal. A técnica utilizada no exame tomográfico foi de $140 \mathrm{~mA}$ e $120 \mathrm{Kvp}$, com filtro adequado para a visibilização dos tecidos ósseos 
e outro para os moles. A espessura do corte e o incremento foram de $2 \mathrm{~mm}$ no escaneamento tomográfico do primeiro posicionamento e de $1 \mathrm{~mm}$ no segundo.

O equipamento de radiologia utilizado no experimento é pertencente ao modelo Ezy rad. $125 \mathrm{Kve}$ o aparelho tomográfico empregado pertence ao modelo SCT-7800CT, ambos da Shimadzu. As imagens tomográficas foram avaliadas utilizando-se o software eFilm Workstation $^{\mathrm{TM}}$ 2.1.2.

Primeiramente, nas radiografias e tomografias pós-operatórias, foi analisado o posicionamento dos componentes acetabular não cimentado e femoral cimentado. Dentro desse item, foi avaliada a articulação dos implantes acetabulares e femorais, sendo esta classificada como adequada ou inadequada (em casos de luxações). O implante femoral foi analisado também quanto à ausência ou presença de deformidades angulares, podendo este ser classificado como normal, varo ou valgo.

Para a avaliação da inter-relação da cabeça femoral protética e do componente acetabular metálico, ao exame tomográfico, foram criados cinco pontos de mensuração da distância entre essas duas estruturas, que foram considerados como equivalentes à espessura do componente acetabular de polietileno de alta densidade, definida como uma imagem hipoatenuante entre essas duas estruturas hiperatenuantes (componentes metálicos). Esses cinco pontos de mensuração foram realizados nas imagens obtidas em plano transversal, sendo fixados em: ponto um (P1), extremidade de contato dorsal (dorsolateral); ponto dois (P2), ponto dorsomedial; ponto três (P3), ponto central; ponto quatro (P4), ventromedial; e ponto cinco (P5), extremidade ventral (ventrolateral) (Fig. 1).

Nas imagens tomográficas transversais, avaliouse de forma qualitativa a presença e o preenchimento do canal femoral pelo cimento ósseo, visibilizado tomograficamente como uma imagem amorfa hiperatenuante adjacente ao implante no interior do canal. Para tal, foram criados cinco pontos predeterminados de avaliação (AC1 - AC5): o ponto 1 (AC1), que representa $\mathrm{o}$ ponto mais proximal, foi determinado pelo ponto de imediato contato da base do implante com o leito ósseo; o ponto 2
(AC2), determinado pela equivalência da altura do trocanter menor do fêmur; o ponto 3 (AC3), que representa uma distância distal ao ponto 2 (AC2), determinada pela distância entre o $\mathrm{AC} 1 \mathrm{e}$ o AC2; o ponto 4 (AC4), que é representado pela extremidade distal do implante; e o ponto 5 (AC5), localizado a $1 \mathrm{~cm}$ distalmente ao AC4 (Fig. 2). O grau de preenchimento do canal femoral com o cimento ósseo foi classificado como: não preenchido; pobremente preenchido; razoavelmente preenchido; bem preenchido; e totalmente preenchido.

Ao exame radiográfico, o cimento ósseo foi analisado somente quanto à sua presença ou ausência, sendo considerado existente quando uma área amorfa de radiodensidade elevada não habitual era visibilizada ao redor do implante no canal femoral. $\mathrm{O}$ aspecto radiográfico do cimento ósseo também foi analisado, sendo este considerado homogêneo ou heterogêneo, na evidência de uniformidade ou não do cimento ósseo, respectivamente.

A presença de radiotransparências ao exame radiográfico e de hipoatenuações à tomografia, na interface prótese-osso dos componentes acetabulares e nas demais regiões, foi analisada no presente estudo; e quando identificadas, estas foram mensuradas. A ocorrência de reações ósseas proliferativas coxofemorais também foi avaliada por ambas as técnicas imaginológicas.

Todos os animais foram submetidos a uma avaliação clínica final da função da articulação coxofemoral por meio da adaptação da escala descrita por Massat e Vasseur (1994) e Guerrero e Montavom (2009), após os três anos de avaliação pós-operatória (M2). Essa escala classifica $o$ resultado final em excelente (ausência de dor, amplitude de movimento articular normal e ausência de claudicação), boa (ausência de dor na manipulação articular, limitação leve de amplitude de movimentos articulares, apoio clinicamente normal do membro), razoável (dor moderada na manipulação articular, amplitude de movimento reduzida, atrofia muscular moderada, claudicação intermitente ou persistente), pobre (dor facilmente detectável na palpação, amplitude de movimento severamente reduzida, atrofia muscular substancial, claudicação severa frequentemente sem apoio) e falha (implantes removidos). 


\section{Avaliação radiográfica...}

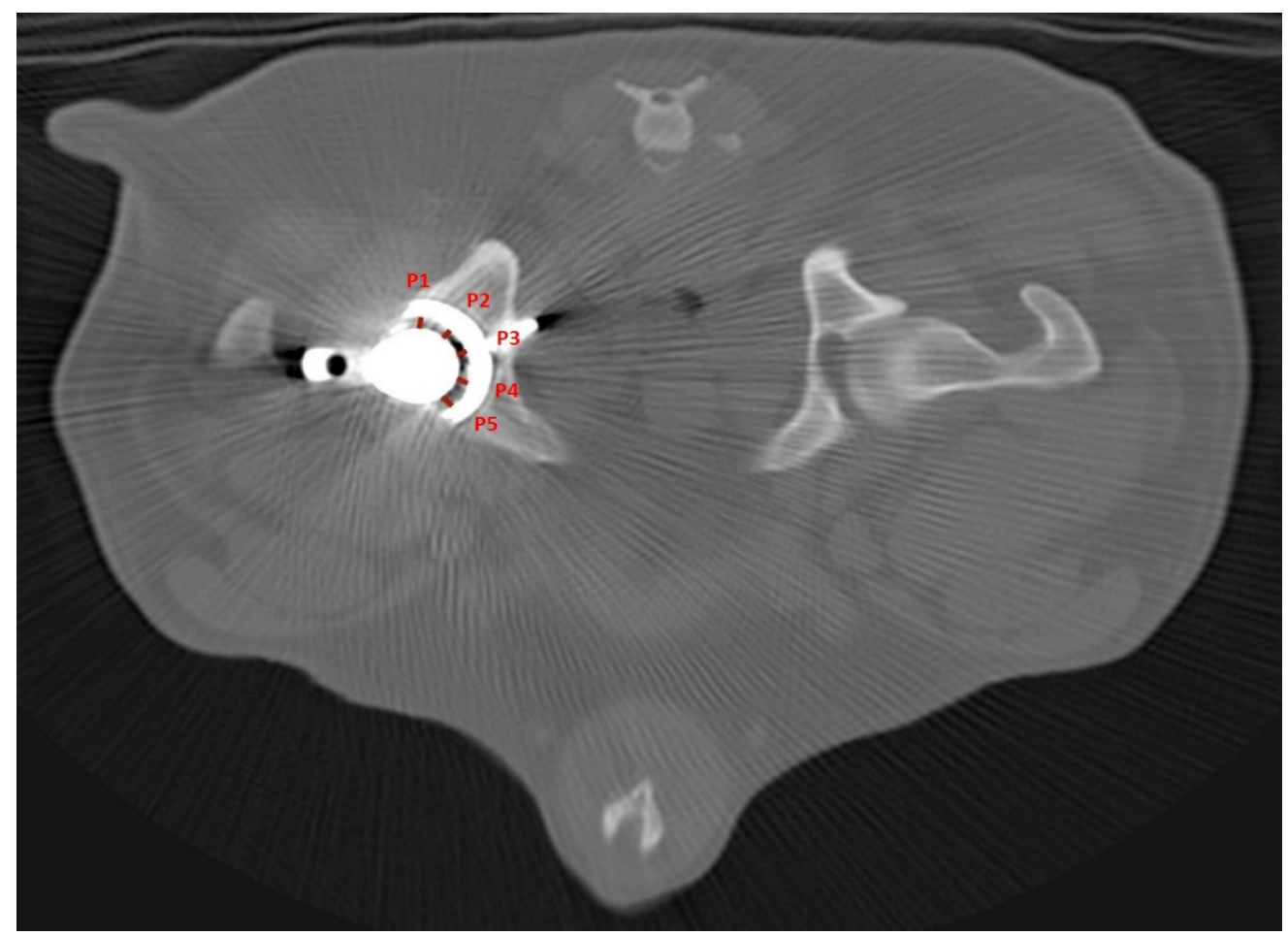

Figura 1. Imagem tomográfica transversal ilustrando a avaliação da distância entre a cabeça femoral protética e o componente acetabular metálico, mensurada em cinco pontos predeterminados: ponto um (P1), extremidade de contato dorsal (dorsolateral); ponto dois (P2), ponto dorsomedial; ponto três (P3), ponto central; ponto quatro (P4), ventromedial; e ponto cinco (P5), extremidade ventral (ventrolateral).

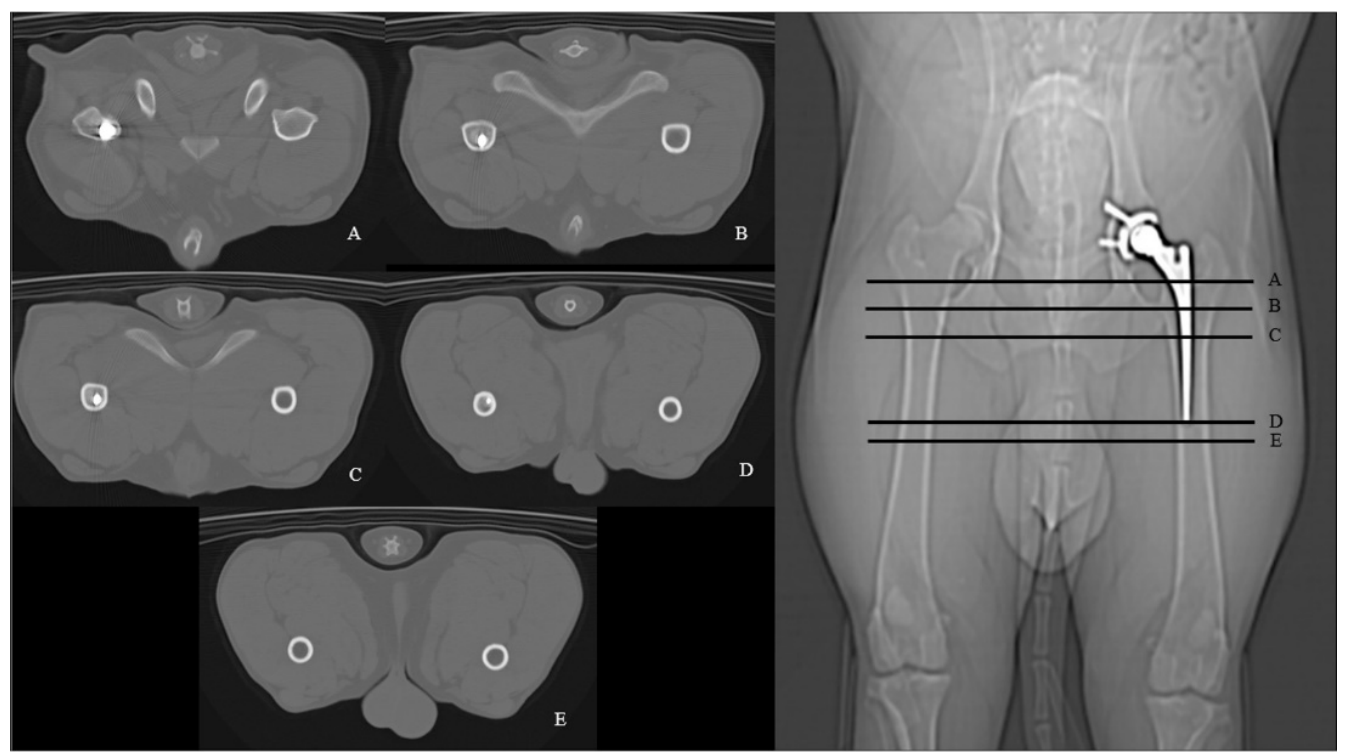

Figura 2. Imagens tomográficas ilustrando os cinco pontos predeterminados para a avaliação subjetiva do cimento ósseo no canal femoral, sendo (A) o ponto $\mathrm{AC}$, determinado pelo ponto de imediato contato da base do implante com o leito ósseo; (B) o ponto AC2, determinado pela equivalência da altura do trocanter menor do fêmur; (C) o ponto $\mathrm{AC} 3$, que representa uma distância distal ao ponto $\mathrm{AC} 2$, determinada pela distância entre o AC1 e o AC2; (D) o ponto AC4, representado pela extremidade distal do implante; e o ponto AC5, localizado a $1 \mathrm{~cm}$ distalmente ao AC4. 


\section{RESULTADOS}

Ao M1, tanto à avaliação radiográfica como tomográfica, todos os animais apresentaram posicionamento adequado do componente protético acetabular não cimentado, sendo observada redução articular adequada com bom encaixe dos componentes protéticos. A haste femoral, no entanto, apresentou-se centralizada em apenas um cão $(\mathrm{C} 1)$ e posicionamento varo nos demais.

A avaliação da inter-relação entre a cabeça femoral protética e o componente acetabular metálico por meio da mensuração tomográfica da distância entre essas duas estruturas ao M1 revelou os seguintes valores para os cães $\mathrm{C} 1$ $(\mathrm{P} 1=0,1 ; \mathrm{P} 2=0,1 ; \mathrm{P} 3=0,2 ; \mathrm{P} 4=0,2 ;$ e $\mathrm{P} 5=0,5), \mathrm{C} 2$ $(\mathrm{P} 1=0,1 ; \mathrm{P} 2=0,1 ; \mathrm{P} 3=0,1 ; \mathrm{P} 4=0,2 ;$ e $\mathrm{P} 5=0,1), \mathrm{C} 3$ $(\mathrm{P} 1=0,1 ; \mathrm{P} 2=0,1 ; \mathrm{P} 3=0,1 ; \mathrm{P} 4=0,1 ;$ e $\mathrm{P} 5=0,2), \mathrm{C} 4$ $(\mathrm{P} 1=0,1 ; \mathrm{P} 2=0,1 ; \mathrm{P} 3=0,1 ; \mathrm{P} 4=0,1 ; \mathrm{e} 5=0,2), \mathrm{C} 5$ $(\mathrm{P} 1=0,1 ; \mathrm{P} 2=0,2 ; \mathrm{P} 3=0,2 ; \mathrm{P} 4=0,3 ;$ e $\mathrm{P} 5=0,2)$, e $\mathrm{C} 6(\mathrm{P} 1=0,1 ; \mathrm{P} 2=0,1 ; \mathrm{P} 3=0,1 ; \mathrm{P} 4=0,1 ;$ e $\mathrm{P} 5=0,1)$.

Ao redor do componente femoral, notou-se, ao exame radiográfico, uma fina camada de cimento na sua interface com o leito ósseo, de aspecto homogêneo em toda a sua extensão, desde o platô até a extremidade distal do implante em todos os animais avaliados. Já à avaliação tomográfica do manto de cimento ósseo, pode-se visibilizar a sua presença nos pontos AC2, AC3, AC4 e AC5 em todos os animais; entretanto, o preenchimento se mostrou razoavelmente preenchido em alguns dos pontos de avaliação. No ponto $\mathrm{ACl}$ não foi possível realizar a presente avaliação devido à dificuldade de visibilização do material hiperatenuante correspondente ao cimento ósseo, uma vez que nessa região observa-se a presença de osso compacto com densidade similar.

Quanto à presença de radiotransparências, ao exame radiográfico foi verificada a uma linha delgada radioluscente focal ao redor do componente acetabular não cimentado em todos os cães, na interface entre as superfícies óssea e metálica. A espessura dessa linha não ultrapassou $1 \mathrm{~mm}$ em toda a sua extensão. De maneira semelhante, foi verificada, ao exame tomográfico, uma linha hipoatenuante contínua e regular ao redor do implante acetabular não cimentado, não maior que $1 \mathrm{~mm}$ de espessura (Fig. 3). Em um cão foi observada uma área de reabsorção óssea ao redor da base de um dos parafusos (craniodorsal), entretanto não foram evidenciados sinais de frouxidão ou soltura do referido componente.

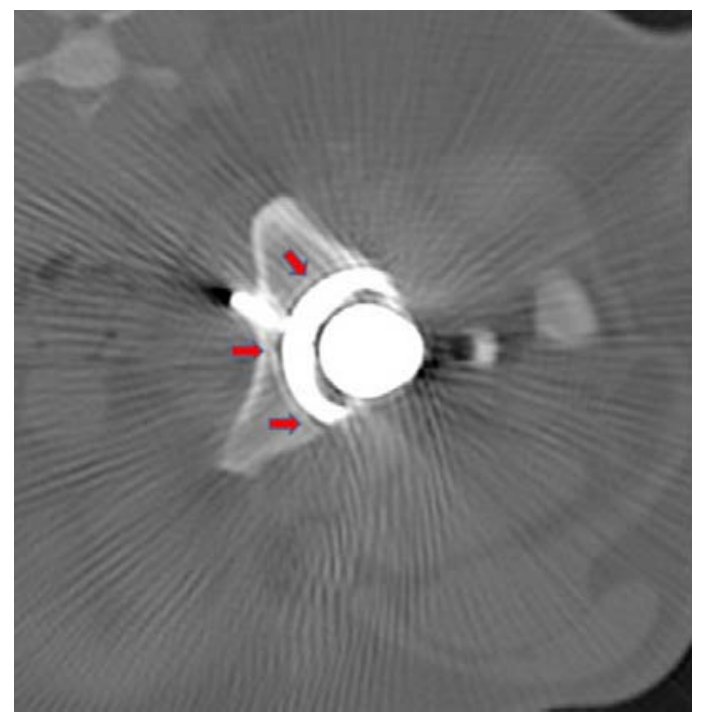

Figura 3. Imagem tomográfica em plano transversal e janela óssea ilustrando a delgada linha radioluscente focal ao redor do componente acetabular não cimentado (setas).

Ao M2, tanto à avaliação radiográfica como tomográfica, não foram verificadas alterações quanto ao posicionamento dos componentes protéticos femoral cimentado e acetabular não cimentado, sendo observada redução articular adequada com bom encaixe dos referidos componentes.

Os aspectos relacionados à avaliação da interface cimento-osso e cimento-prótese, bem como da interface prótese-osso (no caso do componente acetabular), mantiveram-se inalterados ao $\mathrm{M} 2$, tanto ao exame radiográfico como tomográfico, não sendo evidenciadas outras alterações proliferativas ou de reabsorção óssea.

A avaliação clínica final aos três anos de pósoperatório foi realizada e em todos os cães a função da articulação coxofemoral foi classificada como "excelente".

\section{DISCUSSÃO}

A avaliação pós-operatória das artroplastias é imprescindível na determinação do posicionamento dos componentes protéticos e na 
detecção precoce de potenciais complicações (Young e Schiller, 1992; Hanson et al., 2006). Tal avaliação, normalmente realizada por meio de exame radiográfico, de fato possibilita a visibilização da maioria das complicações secundárias ao procedimento de artroplastia total coxofemoral, mesmo aquelas de início assintomático ou muito lento, tais como retroversão da haste femoral, falhas de preenchimento do canal medular femoral, áreas de radioluscência tanto em região do cimento ósseo quanto na interface entre o osso e os implantes, e afundamento da haste femoral no canal medular (Young e Schiller, 1992; Palmisano et al., 2003; Bergh et al., 2006).

No entanto, o exame radiográfico possui algumas limitações e não pode ser utilizado com acurácia para se determinar o posicionamento e a estabilidade dos implantes (Jehn e Manley, 2002; Bergh et al., 2006; Iwata et al., 2008). Já o exame tomográfico por meio de tomografia computadorizada possibilita a aquisição de imagens sem sobreposições e, dessa forma, permite a mensuração e a determinação do posicionamento dos componentes protéticos com maior precisão em relação ao exame radiográfico (Ginja et al., 2007; Kjellberg et al., 2012; Goldvasser et al., 2012).

Tal superioridade da tomografia computadorizada em relação ao exame radiográfico se reflete em menor possibilidade de erro à mensuração, tanto interobservador quanto intraobservador, de áreas de reabsorção óssea ou da espessura de determinados componentes, como a cúpula de polietileno e/ou o componente metálico acetabular (Vandenbussche et al., 2010; Goldvasser et al., 2012).

Embora a tomografia computadorizada apresente maior acurácia quanto à determinação do posicionamento dos componentes protéticos (Goldvasser et al., 2012), no presente estudo, tanto ao exame tomográfico quanto radiográfico, não foram observadas alterações no que se refere ao posicionamento dos implantes, interpretado como a ausência de migração deles.

Ainda quanto ao posicionamento dos componentes protéticos, o exame tomográfico permitiu a obtenção de imagens semelhantes às adquiridas ao exame radiográfico, porém com melhor definição das estruturas ósseas e sua interface com os tecidos moles periarticulares e implantes, tornando possível, assim, a quantificação de alterações periprotéticas e a mensuração das relações articulares. Tais avaliações são pouco descritas para este fim, mesmo apesar da necessidade de uma melhor definição das relações articulares em pacientes submetidos à artroplastia coxofemoral (Ginja et al., 2007; Schmidt et al., 2012) e especialmente em medicina veterinária devido à grande variação racial entre a espécie canina. A conformação das estruturas ósseas que compõem a articulação coxofemoral e a relação destas com os tecidos moles periarticulares estão sujeitas a aspectos raciais, o que torna um grande desafio estabelecer as alterações periprostéticas e mensurar as relações articulares para cada padrão racial (Nelson et al., 2007). A melhor definição das imagens tomográficas torna possível, portanto, estabelecer tais padrões e compreende outra vantagem da técnica em relação à avaliação radiográfica.

A centralização da haste femoral protética foi adequadamente avaliada no presente estudo pelos exames radiográfico e tomográfico. Essa avaliação, no entanto, está mais bem descrita na literatura veterinária por meio do exame radiográfico e está sujeita à conformação femoral proximal (Jehn e Manley, 2002; Jehn et al., 2003). Apesar disso, a tomografia computadorizada também pode ser utilizada para a avaliação de tal parâmetro, fundamental na determinação de fatores de risco para a ocorrência de complicações em potencial, como a ocorrência de reabsorção óssea e fraturas (Jehn et al., 2003).

Com relação à mensuração dos componentes protéticos, o exame tomográfico permitiu a mensuração da espessura do componente acetabular de polietileno, não passível de mensuração por meio do exame radiográfico devido à sobreposição de imagens. A avaliação da interface entre o componente acetabular metálico e a cabeça protética determinou indiretamente a espessura do polietileno de alta densidade, o que pode fornecer informações importantes a respeito do desgaste dessa camada. Ainda assim, essa avaliação não é considerada fundamental na medicina veterinária, visto que as próteses não apresentariam desgaste suficiente para torná-la uma preocupação. Por outro lado, as artroplastias do quadril em pacientes humanos 
frequentemente necessitam de revisão para a substituição do componente acetabular ou de polietileno devido ao desgaste contínuo destes (McMinn et al., 2012).

Ainda quanto à mensuração do componente protético, o exame tomográfico permitiu também a identificação e a mensuração de linhas hipoatenuantes ao redor dos implantes. De fato, a tomografia computadorizada de alta resolução possibilita a reconstrução tridimensional das imagens, sendo este considerado o método ouro para a análise das áreas de reabsorção óssea ao redor dos implantes protéticos (Goldvasser et al., 2012). Adicionalmente, permite a obtenção de imagens multiplanares sem sobreposições, que diminuem a probabilidade de discretas linhas hipoatenuantes não serem visibilizadas, bem como a utilização de janelamento ósseo para melhor caracterização das estruturas periprostéticas.

O estudo dessas linhas nas interfaces dos implantes é fundamental para se predizer a soltura ou o afrouxamento dos componentes protéticos (Bergh et al., 2004; Hanson et al., 2006; Suh et al., 2013) e, uma vez que a tomografia computadorizada se mostre mais específica na sua determinação (Suh et al., 2013), assim como verificado no presente estudo, pode-se considerá-la vantajosa em relação ao exame radiográfico.

De origem variada, a soltura de um implante é a complicação mais frequente em pacientes humanos e caninos e normalmente decorre da formação de debris na interface osso-prótese ou osso-cimento (Suh et al., 2013). Esses debris, por sua vez, são formados do micromovimento da prótese ou do excesso de carga no membro recém-operado e causam uma resposta inflamatória local com consequente osteólise (Liska e Doyle, 2013; Suh et al., 2013).

De acordo com a literatura, a soltura de um implante é caracterizada pela presença de uma linha radioluscente/hipoatenuante ao seu redor, com espessura superior a $1 \mathrm{~mm}$ ou de crescimento progressivo (Young e Schiller, 1992; Hanson et al., 2006). No presente estudo, não foi verificada a soltura dos componentes protéticos acetabular e femoral ao longo do período de avaliação, fato que demonstra uma técnica satisfatória de cimentação no caso do componente femoral (Mostafa et al., 2012).

A cimentação do componente femoral no presente estudo foi considerada boa a excelente, de acordo com a classificação radiográfica proposta por Frankel et al. (2004), sendo caracterizada pela presença de uma camada de cimento ósseo ao redor do referido implante (Massat e Vasseur, 1994). Salienta-se, no entanto, não haver modelos propostos para a avaliação da cimentação por meio do exame tomográfico que, no presente estudo, permitiu a determinação com melhor precisão da quantidade e a distribuição do cimento nos diversos cortes obtidos ao longo do eixo ósseo femoral. Tal vantagem do exame tomográfico possibilita a detecção mais acurada de possíveis falhas de cimentação e, portanto, este pode ser considerado um melhor parâmetro de controle para a ocorrência de eventuais solturas.

Embora a tomografia computadorizada apresente aparente superioridade ao prover imagens sem sobreposições, elucidando detalhes importantes na avaliação das artroplastias coxofemorais, algumas complicações relacionadas à formação de artefatos de imagem puderam ser verificadas no presente estudo. Esses artefatos originaram-se da prótese metálica, o que inviabilizou a avaliação de algumas imagens tomográficas adquiridas em plano dorsal, uma vez que uma extensa área metálica da prótese pôde ser verificada em alguns cortes.

Adicionalmente, segundo Katz (2006) e, mais recentemente, Kjellberg et al. (2012), o exame radiográfico pode subestimar as alterações osteoartríticas devido à sobreposição de imagens e, por isso, apresentar menor acurácia quando comparado à tomografia computadorizada (Jehn e Manley, 2002; Bergh et al., 2006; Iwata et al., 2008). Devido a isso, o exame tomográfico possibilitaria determinar de maneira mais fidedigna o resultado funcional em longo prazo (Suh et al., 2013). No presente estudo, tanto o exame radiográfico quanto o tomográfico, além da avaliação clínica e ortopédica, permitiram determinar o resultado funcional excelente que a técnica híbrida proporcionou aos membros operados, encorajando, assim, o seu desenvolvimento e difusão no Brasil. 


\section{CONCLUSÕES}

A avaliação tomográfica proporcionou imagens semelhantes às obtidas à avaliação radiográfica, porém com melhor definição e detalhamento das estruturas ósseas e do posicionamento dos componentes protéticos, além de mensurar interrelações estruturais. Adicionalmente, o exame tomográfico mostrou-se útil na avaliação das articulações protéticas, com a vantagem de adquirir imagens sem sobreposições, permitindo a identificação e a quantificação de alterações periprotéticas que, por sua vez, indicam a ocorrência de anormalidades nas articulações protéticas. No entanto, observou-se nas imagens tomográficas a ocorrência de diversos artefatos lineares de alta densidade originados da prótese metálica, o que prejudicou parcialmente a avaliação. Essa ocorrência foi especialmente observada nas imagens em plano dorsal, uma vez que uma extensa área metálica da prótese pôde ser verificada em alguns cortes tomográficos, levando, dessa forma, a uma acentuada produção de artefatos.

\section{AGRADECIMENTOS}

À Fapesp, pelo apoio concedido (Processos 2010/20635-2 e 2012/12895-0).

\section{REFERÊNCIAS}

BERGH, M.S.; GILLEY, R.S.; SHOFER, F.S. et al. Complications and radiographic findings following cemented total hip replacement: a retrospective evaluation of 97 dogs. Vet. Comp. Orthop. Traumatol., v.19, p.172-179, 2006.

BERGH, M.S.; MUIR, P.; MARKEL, M.D.; MANLEY, P.A. Femoral bone adaptation to unstable long-term cemented total hip arthroplasty in dogs. Vet. Surg., v.33, p.238-245, 2004.

CHANG, C.Y.; HUANG, A.J.; PALMER, W.E. Radiographic evaluation of hip implants. Semin. Musculoskelet. Radiol., v.19, n.1, p.12-20, 2015.

FRANKEL, D.J.; PLUHAR, E.; SKURLA, C.P. Radiographic evaluation of mechanically tested cemented total hip arthroplasty femoral components retrieved post-mortem. Vet. Comp. Orthop. Traumatol., v.17, p.216-24, 2004.
GINJA, M.M.D.; GONZALO-ORDEN, J.M.; JESUS, S.S.; SILVESTRE, A.M. et al. Measurement of the femoral neck anteversion angle in the dog using computed tomography. Vet. J., v.174, p.378-383, 2007.

GOLDVASSER, D.; NOZ, M.E.; MAGUIRE JR, G.Q. et al. A new technique for measuring wear in total hip arthroplasty using computed tomography. J. Arthroplasty, v.27, p.1636-1640, 2012.

GUERRERO, T.G.; MONTAVON, P.M. Zurich cementless total hip replacement: retrospective evaluation of 2 nd generation implants in 60 dogs. Vet. Surg., v.38, p.70-80, 2009.

HANSON, S.P.; PECK, J.N.; BERRY, C.R. et al. Radiographic evaluation of the Zurich cementless total hip acetabular component. Vet. Surg., v.35, p.550-558, 2006.

IWATA, D.; BROUN, H.C.; BLACK, A.P. et al. Total hip arthroplasty outcomes assessment using functional and radiographic scores to compare canice systems. Vet. Comp. Orthop. Traumatol., v.21, p.221-230, 2008.

JEHN, C.T.; BERGH, M.S.; MANLEY, P.A. Orthogonal view analysis for evaluating the femoral component position of total hip implants in dogs using postoperative radiographs. Vet. Surg., v.32, p.134-141, 2003.

JEHN, C.T.; MANLEY, P.A. The effects of femur and implant position on the radiographic assessment of total hip femoral implants in dogs. Vet. Surg., v.31, p.349-357, 2002.

KATZ, J.N. Total joint replacement in osteoarthritis. Best Pract. Res. Clin. Rheumatol., v.20, p.145-153, 2006.

JELLBERG, M.; AL-AMIRY, B.; ENGLUND, E. et al. Measurement of leg length discrepancy after total hip arthroplasty: the reliability of plain radiographic method compared to CTscanogram. Skeletal Radiol., v.41, p.187-191, 2012.

LASCELLES, B.D.; FREIRE, M.; ROE, S.C.; DEPUY, V. et al. Evaluation of functional outcome after BFX total hip replacement using a pressure sensitive walkway. Vet. Surg., v.39, p.71-77, 2010. 
LISKA，W.D.; DOYLE， N.D. Total joint arthroplasty: surgery and rehabilitation. In: SCIENCE WEEK, 2013. Proceedingas... [Brisbane, Queensland]: The Australian and New Zealand College of Veterinary Scientists, 2013. p.82-88.

MASSAT, B.J; VASSEUR, P.B. Clinical and radiographic results of total hip arthroplasty in dogs: 96 cases (1986-1992). J. Am. Vet. Med. Assoc., v.205, p.448-454, 1994.

MCMINN, D.J.W.; SNELL, K.I.E.; DANIEL, J.; TREACY, R.B.C. et al. Mortality and implant revision rates of hip arthroplasty in patients with osteoarthritis: registry based cohort study. $\mathrm{Br}$. Med. J., v.344, p.2-19, 2012.

MINTO, B.W.; BRANDÃO, C.V.S.; PEREIRA, G.J.C.; CAMPAGNOL, D. et al. Modular hybrid total hip arthroplasty: experimental study in dogs. Acta Vet. Scand., v.53, p.2-6, 2011.

MOSTAFA, A.A.; DRUEN, S.; NOLTE, I.; WEFSTAEDT, P. Radiographic evaluation of early periprosthetic femoral bone contrast and prosthetic stem alignment after uncemented and cemented total hip replacement in dogs. Vet. Surg., v.41, p.69-77, 2012.
NELSON, L.L.; DYCE, J.; SHOTT, S. Risk factors for ventral luxation in canine total hip replacement. Vet. Surg., v.36, p.644-653, 2007.

PALMISANO, M.P.; DYCE, J.; OLMSTEAD, M.L. Extraosseous cement granuloma associated with total hip replacement in 6 dogs. Vet. Surg., v.32, p.80-90,2003.

SCHMIDT, R.; KRESS, A.M.; NOWAK, M. et al. Periacetabular cortical and cancellous bone mineral density loss after press-fit cup fixation: a prospective 7-year follow-up. J. Arthroplasty, v.27, p.1358-1363, 2012.

SUH, D.H.; HAN, S.B.; YUN, H.H. et al. Characterization of progression of pelvic osteolysis after cementless total hip arthroplasty: computer tomographic study. J. Arthroplasty, v.28, p.1851-1855, 2013.

VANDENBUSSCHE, E.; SAFFARINI, M.; HANSEN, U. et al. Measurement of femoral head penetration in polyethylene using a 3dimensional CT-scan technique. Acta Orthop., v.81, p.563-569, 2010.

YOUNG, D.J.; SCHILLER, R.A. Radiographic criteria for evaluation of uncemented total hip replacement in dog. Vet. Surg., v.21, p.88-98, 1992. 